Baeza Franco, L.A y Parra Acosta, H. (2021). La responsabilidad social de la Facultad de Medicina y Ciencias Biomédicas y, su relación con la visión, misión y formación médica. Revista de Investigación Educativa, 39(2), 553-570.

DOI: http://dx.doi.org/10.6018/rie.448101

\title{
La responsabilidad social de la Facultad de Medicina y Ciencias Biomédicas y, su relación con la visión, misión y formación médica
}

\section{The social accountability of the Faculty of Medicine and Biomedical Sciences and its relationship with the vision, mission and medical education}

\author{
Laura Artemisa Baeza Franco* y Haydeé Parra Acosta**1 \\ * Facultad de Medicina y Ciencias Biomédicas. Universidad Autónoma de Chihuahua. (México) \\ ** Departamento de Investigación Educativa. Facultad de Medicina y Ciencias Biomédicas. Universidad Autónoma de \\ Chihuahua (México).
}

\begin{abstract}
Resumen
La responsabilidad social universitaria (RSU) es una política educativa de consciencia de los impactos académicos de los universitarios en la sociedad, los cuales son indispensables para la innovación de la educación y el fortalecimiento de las estructuras sociales. El objetivo de este estudio fue valorar la percepción que tienen los estudiantes, personal académico, directivos y jefes de enseñanza, respecto a la responsabilidad social y determinar su relación con la visión, misión y formación médica. Se realizó un estudio transversal tipo correlacional mediante la aplicación de un cuestionario. Se determinaron los aspectos de la responsabilidad social más valorados por estudiantes, personal académico, directivos y jefes de enseñanza, se establecieron diferencias significativas de la responsabilidad social en estos cuatro grupos con un nivel de significancia de .005 y se observaron relaciones significativas con los aspectos de la visión, misión y formación médica. En este estudio se resaltó, que los aspectos más percibidos de la responsabilidad social permiten valorarla desde un enfoque socio formativo que incide en la innovación educativa con la implementación de unidades de aprendizaje y programas de responsabilidad social. Esta investigación aporta al estado del conocimiento sobre la responsabilidad social al demostrar que
\end{abstract}

Correspondencia: Haydeé Parra Acosta, hparra05@hotmail.com. Circuito Universitario. Campus II. C.P. 31125 
la percepción que tienen los estudiantes y el personal académico sobre la responsabilidad social incide en la formación médica, la misión y visión de la facultad.

Palabras clave: responsabilidad social; formación médica; percepción; evaluación formativa.

\section{Abstract}

University social accountability is an educational policy of awareness regarding the academic impact of university students in society, which is essential for the innovation of education and the strengthening of social structures. The objective of this study was to assess the perception that students, academic personnel, executive boards, and headteachers have regarding social accountability and determine its relationship with the faculty's vision, mission, and medical education. A cross-sectional, correlational study was carried out by applying a questionnaire. The aspects of social accountability most valued by students, academic personnel, executives, and headteachers were determined, while significant differences in social accountability were established in these four groups with a significance level of .005. Also, significant relationships were observed with aspects of the faculty's vision, mission, and medical education. In this study, it was highlighted that the most perceived aspects of social accountability allow it to be assessed from a socio-formative approach that affects educational innovation with the implementation of learning units and social accountability programs. This research contributes to the state of knowledge about social accountability by demonstrating that the perception that students and academic personnel have about it affects medical education, as well as the mission and the vision of the faculty.

Key words: social accountability; medical education; perception; formative evaluation.

\section{Introducción}

La responsabilidad social es la consciencia del impacto que tienen las acciones en la sociedad del futuro. Inició como una ideología, después, se observó como una tendencia de procurar el bien de la comunidad. Pese a que se identifica principalmente en el contexto empresarial, se ha implementado a través del tiempo en el sector educativo (Flórez Guzmán et al., 2016).

En la educación, la responsabilidad social se introdujo como una política con enfoque de cuidado y consciencia de los impactos administrativos y académicos en los procesos de la universidad (Vallaeys \& Álvarez Rodríguez, 2019). La intención es que los universitarios se relacionen con su entorno en un constante diálogo con la sociedad. La universidad debe ser líder en la generación de conocimientos de alcance global (Martínez-Domínguez \& Porto-Pedrosa, 2018).

En la literatura se mencionan diferentes perspectivas de la Responsabilidad Social Universitaria (RSU). Se hace referencia al enfoque consecuencialista, contractual y prospectivo. Pese a los diferentes enfoques, las acciones que caracterizan a la RSU van más allá del altruismo (Gaete Quezada, 2016).

La formación universitaria debe profundizar en la preparación profesional y aportar una formación integral y socioformativa del estudiante, para que contribuya a la 
solución de problemas en su comunidad con idoneidad y compromiso ético (García Ramos et al., 2016; Tobón, 2017).

En las escuelas de medicina el concepto de responsabilidad social se ha interpretado de diferentes maneras, entre ellas como el mayor deber social que se tiene, por lo que requiere de monitoreo continuo, ya que su evaluación está basada en el impacto de los egresados en la salud de la sociedad (Boelen et al., 2019).

Para guiar el desarrollo de escuelas de medicina socialmente responsables existen diversas iniciativas que han adoptado un plan educativo de medicina basada en la salud de la comunidad. De esta manera, se han comprometido con la sociedad a través de la responsabilidad social (Reeve et al., 2016). En este sentido, algunas escuelas de medicina fueron reconocidas socialmente responsables por miembros de la OMS, siendo estas las que dirigían su educación y actividades a servir a la sociedad en la que se encuentran (Preston et al., 2016).

Así mismo, las escuelas de medicina socialmente responsables cimientan su misión, visión y valores en el acercamiento a la comunidad, promoción de la justicia en salud, uso adecuado de los recursos y búsqueda de los factores sociales que afectan la salud. El enfoque de la educación en la misión social se basa en la consciencia de la responsabilidad que implica ser una institución educativa (Mullan, 2017).

No obstante, la formación médica tiene como prioridad el crecimiento académico de sus egresados, enfocándose a la atención de enfermedades ya instauradas a través de un sistema de salud basado en especialidades médicas y no en atención primaria con educación a la población (Greer Jr et al., 2017). En este contexto, las escuelas de medicina del siglo XXI enfrentan retos, como son: mejorar la prestación de servicios de la salud, atender las prioridades de la sociedad y definir nuevos roles a los profesionales de la salud para incidir en la salud de la población (Boelen \& Woollard, 2011).

La evaluación del impacto de las escuelas de medicina en la salud y su relación con la responsabilidad social se ha realizado a través de diversos estudios. En Irán se realizó un estudio a través de un instrumento que evaluó la responsabilidad social de las escuelas de medicina. Se diseñó una herramienta para medir la formación de los médicos socialmente responsables y su impacto en la salud de la sociedad (Shahram et al., 2019).

En Mashhad, Irán se realizó otro estudio transversal en los centros de salud y se intentó evaluar la RSU por medio del desarrollo de un instrumento. Se observó que las limitaciones de responsabilidad social más identificadas fueron: la necesidad de familiarización de los estudiantes con los centros de salud, las enfermedades comunes y la educación médica basada en la comunidad. Lo cual se relacionó con la falta de conocimiento de las necesidades de salud de la sociedad para poder ofrecer servicios de salud efectivos (Ali et al., 2016).

En Latinoamérica también se observó la falta de instrumentos para la medición del avance en responsabilidad social, ya que la mayoría de los estudios realizados son de países desarrollados con diferentes problemáticas sociales. Por esto, se realizó un instrumento validado por expertos en responsabilidad social de 49 universidades encontradas en 16 países de Latinoamérica con la finalidad de medir el impacto de las escuelas de medicina en la sociedad (Puschel et al., 2020). 
En México se realizó una investigación transversal cuantitativa con el fin de identificar los elementos que conforman a la educación médica. Se aplicó un cuestionario que abarcaba seis dimensiones de análisis, dentro de las cuales se incluía el impacto social. Se determinó que, pese a que todas las escuelas consideraron que sus egresados solventan apropiadamente los problemas de salud existentes, se reconoce la falta de evaluaciones del desempeño de sus egresados con base en el impacto en la salud de las comunidades a las que ellos sirven (Fajardo-Dolci et al., 2019).

Así mismo, en un estudio reciente realizado por Abreu-Hernández et al., se identificaron los retos que las escuelas y facultades de medicina enfrentan con la contingencia de COVID-19. Dentro de ellos se encuentran, la falta de enfoque a la atención primaria de la salud, la segmentación de contenido académico y aprendizaje clínico descoordinado, el enfoque industrializado de la medicina y la generación de investigación centralizada. Estos retos evidencian la necesidad de eliminar los esquemas lineales del aprendizaje para reemplazarlos por estructuras flexibles que permitan una transición de impacto para la salud de todos (Abreu-Hernández et al., 2020).

\section{Definición del problema y justificación}

Existen instrumentos para medir la percepción sobre responsabilidad social que tienen los estudiantes y otros actores de las escuelas de medicina de manera objetiva la responsabilidad social. Sin embargo, aun cuando las percepciones de los universitarios en salud parecen ser favorables para el conocimiento y aplicación de la responsabilidad social, se puede apreciar la falta de evaluaciones objetivas del desempeño de los egresados y cómo impactan en la salud de las comunidades.

En el análisis de diversos artículos se observó, que existen vacíos de información respecto a cómo perciben los directivos, los docentes de la facultades y escuelas de medicina y los responsables académicos de los hospitales, el impacto que tiene la responsabilidad social en la formación médica, así como de las acciones que se realizan desde la facultad de medicina para contribuir en la solución idónea de los problemas de salud que más afectan a la sociedad. También se observó que se carece de estudios sobre la relación que existe entre la responsabilidad social, la visión, misión y formación médica en las facultades de medicina. Si bien, la responsabilidad social es un tema de interés y discusión actual, los artículos publicados toman en cuenta solo, la opinión de los estudiantes. Es así, que surge la necesidad de evaluar la percepción que tienen también directivos, docentes y jefes de enseñanza. Debido a esto, se realizó este estudio para dar respuesta a la siguiente interrogante de investigación:

¿Cómo se percibe la responsabilidad social de la Facultad de Medicina y Ciencias Biomédicas y qué relación muestra con la visión, misión y formación médica?

Esta investigación aporta al estado del conocimiento sobre la responsabilidad social de las escuelas de medicina. Su relevancia radica en que determina su impacto en la formación médica actual, ante los problemas de la sociedad a partir de la percepción de los directivos, docentes y jefes de enseñanza. De igual forma aporta información sobre las áreas de oportunidad que se tienen en la formación médica.

Su contribución práctica es el proceso implementado para valorar el impacto que tienen las acciones formativas en los problemas de salud. Asimismo, su valor teórico 
consiste en la generación de conocimiento novedoso sobre la responsabilidad social que manifiesta la escuela de medicina actualmente y su relación con la visión, misión y formación médica.

Otra cuestión relevante de estudio es su utilidad metodológica al dar a conocer un instrumento validado en su consistencia interna que mide la percepción de la responsabilidad social de la Facultad de Medicina.

\section{Método}

\section{Objetivos}

Objetivo general:

Valorar la percepción que tienen estudiantes, personal académico, directivos y jefes de enseñanza respecto a la responsabilidad social y determinar su relación con la visión, misión y formación médica.

Objetivos específicos:

Describir los aspectos de la responsabilidad social considerados más importantes.

Determinar la relación significativa entre la percepción de la responsabilidad social y la visión, misión y formación médica.

Establecer diferencias significativas entre la percepción que tienen los estudiantes, personal académico, directivos y jefes de enseñanza respecto a la responsabilidad social.

Para cumplir con estos objetivos se realizó un estudio transversal tipo correlacional con enfoque cuantitativo para lograr la recopilación de datos en un momento único y realizar la asociación variables para probar hipótesis con base en la medición numérica y el análisis estadístico (Hernández et al., 2014).

Se aplicó un cuestionario dirigido a estudiantes, personal académico, directivos y jefes de enseñanza respecto a la responsabilidad social de la Facultad de Medicina y Ciencias Biomédicas de la Universidad Autónoma de Chihuahua; que integra cuatro variables complejas: responsabilidad social, formación médica, visión y misión de la facultad. El instrumento fue validado en su consistencia interna y aplicado en el semestre de enero a agosto de 2020 para el procesamiento y análisis de los resultados.

\section{Hipótesis}

Fueron tres hipótesis a contrastar en este estudio:

$\mathrm{H}_{1}$ Los aspectos de la responsabilidad social más valorados refieren a: la implementación de unidades de aprendizaje de formación responsabilidad social, participación con otras instituciones en la detección de problemas de salud, inclusión de los valores de la responsabilidad social en la misión, programa educativo dirigido a la formación de médicos líderes y la adecuación de proyectos de investigación basados en reglamentos.

$\mathrm{H}_{0}$ Los aspectos de la responsabilidad social menos valorados refieren a: la implementación de unidades de aprendizaje de formación en responsabilidad social, participación con otras instituciones en la detección de problemas de salud, inclusión de los valores de la responsabilidad social en la misión, programa educativo dirigido a la formación de médicos líderes y la adecuación de proyectos de investigación basados en reglamentos. 
$\mathrm{H}_{2}$ Existen diferencias estadísticas significativas entre la percepción que tienen los estudiantes, personal académico, directivos y jefes de enseñanza respecto a la responsabilidad social.

$\mathrm{H}_{0}$ No existen diferencias estadísticas significativas entre la percepción que tienen los estudiantes, personal académico, directivos y jefes de enseñanza respecto a la responsabilidad social.

$\mathrm{H}_{3}$ Existe relación significativa entre la percepción de la responsabilidad social que tienen los estudiantes, personal académico, directivos, jefes de enseñanza y la visión, misión y la formación médica.

$\mathrm{H}_{0}$ No existe relación significativa entre la percepción de la responsabilidad social que tienen los estudiantes, personal académico, directivos, jefes de enseñanza y la visión, misión y la formación médica.

\section{Población y Muestra}

La población de estudio corresponde a 2,052 individuos de los cuales 1,607 son estudiantes, 426 docentes, 6 directivos y 13 jefes de enseñanza en la carrera de Médico Cirujano y Partero de la Facultad de Medicina y Ciencias Biomédicas de la Universidad Autónoma de Chihuahua.

Se utilizó la fórmula de William Cochran (en Hernández-Sampieri, 2014) para el cálculo de la muestra inicial: $n=Z^{2}(p \times q) \div e^{2}$; ; En donde " $n$ " es la muestra, " $\mathrm{z}$ " es el nivel de confianza, " $\mathrm{p}$ " es la probabilidad de que ocurra el suceso esperado, " $\mathrm{q}$ " la probabilidad de que no ocurra el proceso esperado y " $\mathrm{e}$ " el margen de error.

Se usó un nivel de confianza del 95\% (1.96), la probabilidad de que ocurra el suceso esperado de $50 \%$ (.5), la probabilidad de que no ocurra el proceso esperado de $50 \%$ (.5) y un margen de error de 5\% (.05). Se obtuvo un tamaño de muestra inicial de 384 individuos.

Se obtuvo una muestra corregida de 324 sujetos con la ecuación:

$$
n=n_{o} \div\left(1+\frac{n_{o}-1}{N}\right)
$$

En donde "N" representa el número de elementos de la población y " $\mathrm{n}_{\mathrm{o}}$ " el tamaño provisional de la muestra.

Esta muestra corregida se estratificó tomando en cuenta el porcentaje correspondiente a cada grupo poblacional, obteniendo así una muestra estratificada de 254 estudiantes, 67 docentes, 1 directivo y 2 jefes de enseñanza que son un total de 324 sujetos.

\section{Instrumento}

El instrumento tipo cuestionario estuvo dirigido a estudiantes, personal académico, directivos y jefes de enseñanza. Integró cuatro variables demográficas: carrera cursada, semestre cursado, edad y sexo en el caso de estudiantes; puesto desempeñado, lugar en donde se desempeña, edad y sexo en el caso del personal académico, directivos y jefes de enseñanza. 
Este cuestionario midió la percepción de responsabilidad social y su relación con la formación médica, la misión y visión a través de cuatro variables complejas las cuales se integraron en preguntas de la siguiente forma:

Tabla 1

Contenido del instrumento dirigido a estudiantes.

\begin{tabular}{lcc}
\hline Variable compleja & Número de preguntas & Preguntas en total \\
\hline Responsabilidad social & $1-27$ & 27 \\
Formación médica & $28-47$ & 20 \\
Misión & $48-53$ & 6 \\
Visión & $54-60$ & 7 \\
\hline
\end{tabular}

Tabla 2

Contenido del instrumento dirigido a personal académico, directivos y jefes de enseñanza.

\begin{tabular}{lcc} 
Variable compleja & Número de preguntas & Preguntas en total \\
\hline Responsabilidad social & $1-26$ & 26 \\
Formación médica & $27-47$ & 21 \\
Misión & $48-53$ & 6 \\
Visión & $54-60$ & 7 \\
\hline
\end{tabular}

Estas preguntas se valoran mediante una escala Likert (del 0 al 4) tomando la siguiente escala de valoración:

Tabla 3

Escala de valoración.

\begin{tabular}{cc}
\hline Escala & Valor \\
\hline $\mathbf{0}$ & Nunca / Deficiente / Nada \\
$\mathbf{1}$ & Pocas veces/ Regular / Poco \\
$\mathbf{2}$ & Algunas veces/ Bueno / Suficiente \\
$\mathbf{3}$ & Frecuente / Muy Bueno / Bastante \\
$\mathbf{4}$ & Siempre/ Excelente / Totalmente \\
\hline
\end{tabular}

Para validar el instrumento en su consistencia interna se envió a dos grupos piloto de 20 personas vía correo electrónico, los cuales debían pertenecer al personal acadé- 
mico y estudiantes de la facultad de medicina. Los integrantes de ambos grupos piloto debían pertenecer al personal académico o cuerpo estudiantil de la facultad. El análisis de consistencia interna se realizó a través del Alpha de Cronbach donde se obtuvo un valor de .98 lo cual indica que es un instrumento altamente confiable.

\section{Procedimiento de recogida y análisis de datos}

Análisis univariado:

Se realizó un análisis exploratorio para valorar si el comportamiento de las variables era normal. Mediante el análisis descriptivo se caracterizó a la población objetivo. Se realizó un análisis descriptivo de medias para identificar a las variables con un valor superior o inferior al intervalo de $\langle X-1 \sigma, X+1 \sigma\rangle$. Con este análisis se determinaron las variables que sobresalen de los límites de normalidad establecidos; denominándose atípico superior o inferior.

Análisis bivariado:

Se establecieron relaciones significativas entre los aspectos de la responsabilidad social que más son percibidos con la visión, misión y formación médica a través de correlación $r$ de Pearson.

Se establecieron comparativos entre estudiantes, personal académico, directivos y jefes de enseñanza por medio de estudio comparativo de ANOVA One way por ser más de dos grupos poblacionales.

\section{Resultados}

\section{Percepciones de la responsabilidad social considerados por el personal académico, directivos y jefes de enseñanza}

En el análisis de medias se observó, de acuerdo a los límites de normalidad a una desviación estándar $(\mathrm{X}+1 \sigma)$, que las variables: Implementan unidades de aprendizaje de formación en responsabilidad social (3.11 .81 ), participan con otras instituciones en la detección de problemas de salud (3.22 \pm 1.02$)$ e incluyen los valores de equidad, calidad, rentabilidad, participación comunitaria y profesionalismo en su misión social $(3.27 \pm .72)$, resaltaron como atípicas superiores a los valores de la media $(2.82+.22=$ 3.04). Lo cual indica que el personal académico, directivos y jefes de enseñanza perciben que frecuentemente se implementa la educación de responsabilidad social, se detectan problemas de salud en conjunto con otras instituciones y se incluyen valores de la responsabilidad social en la misión de su facultad.

Sin embargo, las variables que resultaron como atípicas inferiores a $(X-1 \sigma)$ en el análisis de medias $(2.82-.22=2.60)$ son: Destinan presupuesto al desarrollo de investigaciones $(2.42 \pm 1.01)$, contribuyen a la distribución equilibrada de egresados en las zonas urbanas y rurales $(2.56 \pm 1.21)$, cuentan con herramientas válidas para medir su impacto en la salud de la comunidad $(2.58 \pm .98)$ y cuentan con herramientas válidas para medir la responsabilidad social de sus actividades $(2.59 \pm 1.18)$. Ello muestra que, pese a que los valores son bajos el personal académico, directivos y jefes de enseñanza perciben que es suficiente el presupuesto destinado a la investigación, la contribución 
de distribución equilibrada de los egresados, así como las herramientas para medir el impacto en salud y la responsabilidad social de las actividades (Ver figura 1).

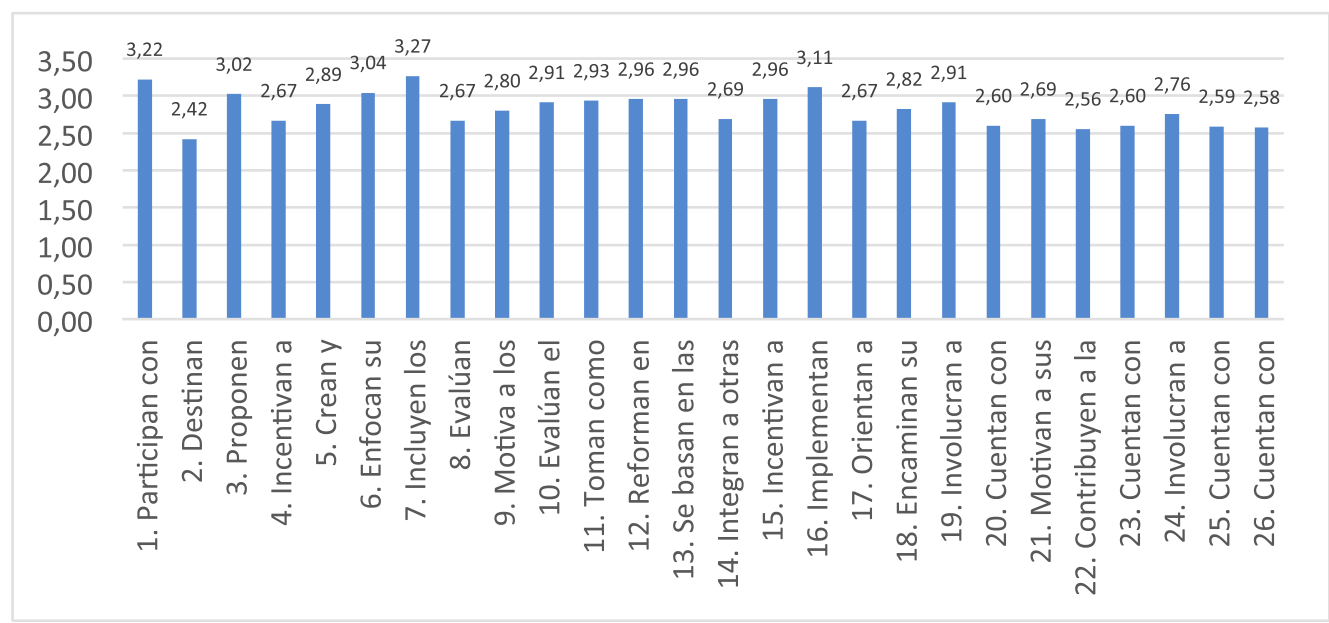

Figura 1. Aspectos de la responsabilidad social más percibidos por personal académico, directivos y jefes de enseñanza.

\section{Percepciones de la responsabilidad social considerados por los estudiantes}

En el análisis de medias se observó que de acuerdo a los límites de normalidad a una desviación estándar $(X+1 \sigma)$, las variables que resaltaron como atípicas superiores a los valores de la media $(2.90+.14=3.03)$ fueron: Implementan unidades de aprendizaje de formación en responsabilidad social (3.10 \pm .95$)$, encaminan su programa educativo a la formación de médicos con capacidades sociales con el fin de que pueda ejercer su liderazgo en las comunidades (3.13 \pm 1.23$)$, incluyen los valores de equidad, calidad, rentabilidad, participación comunitaria y profesionalismo en su misión social (3.14 \pm .92) y evalúan la adecuación de los proyectos de investigación con base en políticas y reglamentos $(3.15 \pm .89)$. Esto indica que los estudiantes perciben que frecuentemente se implementa la educación de responsabilidad social, se forman médicos con capacidad de liderazgo, se incluyen los valores de la responsabilidad social en la misión de su facultad y el apego de los proyectos de investigación a reglamentos.

En cambio, las variables que resultaron como atípicas inferiores a (X-1 $\sigma)$ en el análisis de medias $(2.90-.14=2.76)$ son: Renuevan el plan de estudios en función de las necesidades de la sociedad $(2.60 \pm 1.24)$, incentivan a los estudiantes y profesores a realizar investigaciones que apoyen el desarrollo social $(2.63 \pm 1.13)$ y crean programas en donde se evalúe el impacto en la salud de la sociedad (2.69 \pm 1.09$)$. Ello significa que, pese a que los valores son bajos, los estudiantes perciben como bueno o suficiente, la innovación del plan de estudios en función de las necesidades sociales, la motivación a estudiantes y docentes a realizar investigación, así como a la creación de programas para la evaluación de la salud (Ver figura 2). 


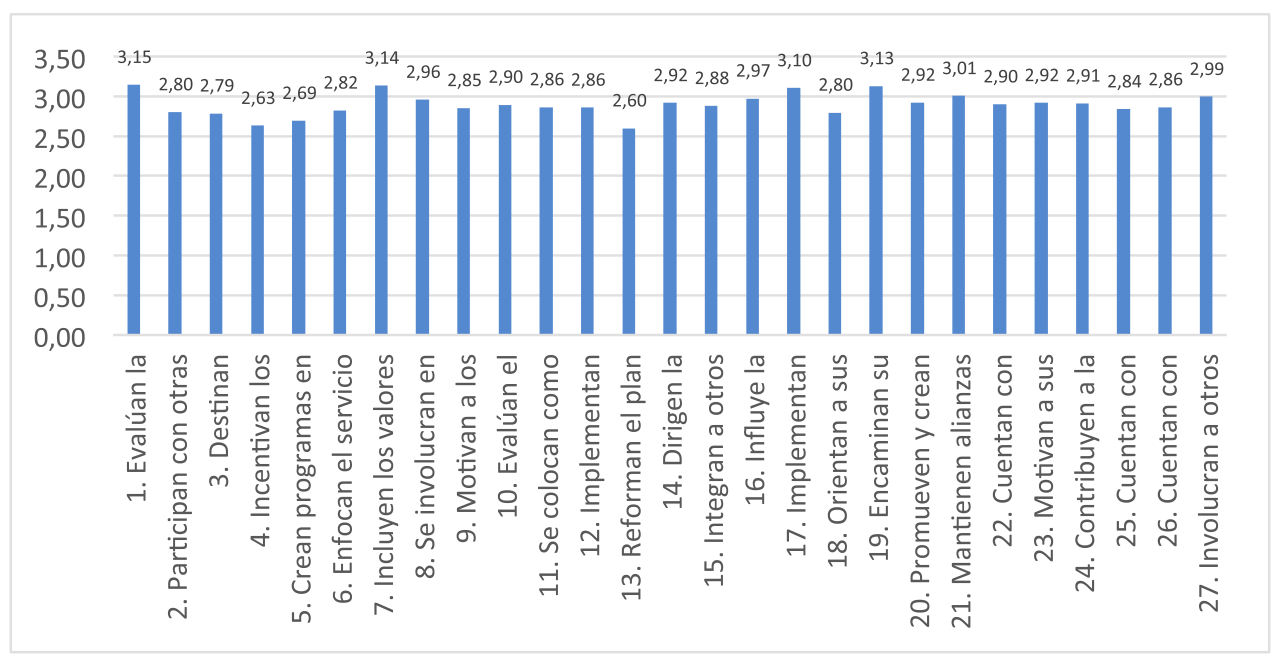

Figura 2. Aspectos de la responsabilidad social más percibidos por estudiantes.

\section{Diferencias significativas entre la percepción que tienen los estudiantes, personal académico, directivos y jefes de enseñanza respecto a la responsabilidad social}

Los resultados del análisis de comparación de medias a través de la técnica One Way Anova mostraron que existen diferencias significativas con una $\mathrm{p}(<) .05$, respecto a la carrera y puesto que desempeñan. Se encontraron diferencias significativas entre estudiantes y personal de salud con respecto a las variables que hacen referencia a la evaluación periódica de las deficiencias de los servicios de salud y contar con planes de seguimiento de los egresados en la comunidad, observándose que son los estudiantes los que más perciben más, la evaluación de deficiencias y el seguimiento de los egresados (Ver tabla 4).

Tabla 4

Percepción de los estudiantes, personal académico, directivos y jefes de enseñanza respecto a responsabilidad social (ANOVA).

\begin{tabular}{|c|c|c|c|c|c|c|c|}
\hline \multirow[t]{2}{*}{$\begin{array}{c}\text { Variable } \\
\text { dependiente }\end{array}$} & \multirow[t]{2}{*}{ HSD Tukey } & & \multirow[t]{2}{*}{$\begin{array}{l}\text { Diferencia de } \\
\text { medias (I-J) }\end{array}$} & \multirow[t]{2}{*}{$\begin{array}{l}\text { Desv. } \\
\text { Error }\end{array}$} & \multirow[t]{2}{*}{ Sig. } & \multicolumn{2}{|c|}{$\begin{array}{c}\text { Intervalo de } \\
\text { confianza al } 95 \%\end{array}$} \\
\hline & & & & & & $\begin{array}{l}\text { Límite } \\
\text { inf. }\end{array}$ & $\begin{array}{l}\text { Límite } \\
\text { sup. }\end{array}$ \\
\hline \multirow{6}{*}{$\begin{array}{l}\text { 8. Evalúan } \\
\text { periódicamente } \\
\text { las deficiencias en } \\
\text { los servicios de } \\
\text { salud? }\end{array}$} & Otro & Docente & -.769 & .428 & .172 & -1.78 & .24 \\
\hline & & Estudiante & $-.958^{*}$ & .404 & .049 & -1.91 & .00 \\
\hline & Docente & Otro & .769 & .428 & .172 & -.24 & 1.78 \\
\hline & & Estudiante & - .189 & .171 & .513 & -.59 & .21 \\
\hline & Estudiante & Otro & $.958^{*}$ & .404 & .049 & 0 & 1.91 \\
\hline & & Docente & .189 & .171 & .513 & -.21 & .59 \\
\hline
\end{tabular}




\begin{tabular}{|c|c|c|c|c|c|c|c|}
\hline \multirow[t]{2}{*}{$\begin{array}{c}\text { Variable } \\
\text { dependiente }\end{array}$} & \multirow[t]{2}{*}{ HSD Tukey } & & \multirow[t]{2}{*}{$\begin{array}{l}\text { Diferencia de } \\
\text { medias (I-J) }\end{array}$} & \multirow[t]{2}{*}{$\begin{array}{l}\text { Desv. } \\
\text { Error }\end{array}$} & \multirow[t]{2}{*}{ Sig. } & \multicolumn{2}{|c|}{$\begin{array}{c}\text { Intervalo de } \\
\text { confianza al } 95 \% \\
\end{array}$} \\
\hline & & & & & & $\begin{array}{l}\text { Límite } \\
\text { inf. }\end{array}$ & $\begin{array}{l}\text { Límite } \\
\text { sup. }\end{array}$ \\
\hline \multirow{6}{*}{$\begin{array}{l}\text { 23. Cuentan } \\
\text { con planes de } \\
\text { seguimiento del } \\
\text { desenvolvimiento } \\
\text { de sus egresados } \\
\text { en la comunidad? }\end{array}$} & Otro & Docente & -1.077 & .482 & .068 & -2.21 & .06 \\
\hline & & Estudiante & $-1.172 *$ & .456 & .029 & -2.25 & -.10 \\
\hline & Docente & Otro & 1.077 & .482 & .068 & -.06 & 2.21 \\
\hline & & Estudiante & -.095 & .193 & .875 & -.55 & .36 \\
\hline & Estudiante & Otro & $1.172^{*}$ & .456 & .029 & .10 & 2.25 \\
\hline & & Docente & .095 & .193 & .875 & -.36 & .55 \\
\hline
\end{tabular}

*. La diferencia de medias es significativa en el nivel .05

\section{Relación entre la percepción de la responsabilidad social que tienen los estudiantes, el personal académico, directivos, jefes de enseñanza y la visión de la facultad}

Se observó relación significativa entre la variable de la responsabilidad social y la participación conjunta con otras instituciones en la detección de problemas de salud respecto a las variables de la visión de la facultad asociadas a fomentar la investigación para generar conocimiento ( $\mathrm{r}$.544) y motivar a estudiantes, docentes y egresados a participar en proyectos de investigación ( $\mathrm{r} .550)$.

Así mismo, se mostró relación significativa entre la inclusión de los principales valores de la responsabilidad social a la misión de la facultad, con las variables de la visión vinculadas con fomentar la investigación para generar conocimiento $(\mathrm{r}$.608) y motivar a estudiantes, docentes y egresados a participar en proyectos de investigación ( $\mathrm{r}$.638). También se encontró relación significativa entre la implementación de unidades de aprendizaje de formación en responsabilidad social, con las variables de la visión de la facultad referidas a fomentar la investigación para generar conocimiento $(\mathrm{r}$.542) y motivar a estudiantes, docentes y egresados a participar en proyectos de investigación ( $\mathrm{r}$.582).

Existe también relación significativa entre el apego del programa educativo a la formación de médicos líderes y las variables de la visión de la facultad sobre fomentar la investigación para generar conocimiento ( $\mathrm{r}$.550) y motivar a estudiantes, docentes y egresados a participar en proyectos de investigación ( $\mathrm{r}$.562) (Ver tabla 5). 
Tabla 5

Correlaciones de Pearson entre variables de responsabilidad social y visión.

\begin{tabular}{|c|c|c|l|c|}
\hline \multicolumn{1}{|c|}{ II. Visión } & $\begin{array}{l}\text { 1. Participan } \\
\text { con otras } \\
\text { instituciones en } \\
\text { la detección de } \\
\text { problemas de } \\
\text { salud }\end{array}$ & $\begin{array}{l}\text { 7. Incluyen los } \\
\text { valores de la } \\
\text { responsabilidad } \\
\text { social en su } \\
\text { misión social }\end{array}$ & $\begin{array}{l}\text { 16. Implementan } \\
\text { unidades de } \\
\text { aprendizaje de } \\
\text { formación en } \\
\text { responsabilidad } \\
\text { social }\end{array}$ & $\begin{array}{l}\text { 18. Encaminan } \\
\text { su programa } \\
\text { educativo a la } \\
\text { formación de } \\
\text { médicos líderes }\end{array}$ \\
$\begin{array}{l}\text { 58. Se fomenta la } \\
\text { participación en } \\
\text { investigación para } \\
\text { generar conocimiento } \\
\text { 59. Se motiva a } \\
\text { estudiantes, egresados } \\
\text { y docentes a participar } \\
\text { en proyectos de } \\
\text { investigación }\end{array}$ & $.544^{*}$ & $.608^{*}$ & $.542^{*}$ & $.550^{*}$ \\
\hline
\end{tabular}

Relación entre la percepción de la responsabilidad social que tienen los estudiantes, el personal académico, directivos, jefes de enseñanza y la misión de la facultad

Se mostró que existe relación significativa entre la difusión de la misión y la inclusión de los principales valores de la responsabilidad social en la misión de la facultad ( $r$.524) e incentivar a docentes y estudiantes a participar en actividades de responsabilidad social (r .549).

También se observó relación significativa entre la formación de profesionales apegados a los valores de la facultad y las variables vinculadas con dirigir el servicio social a la resolución de problemas de salud (r .501) y la determinación de los objetivos de aprendizaje basados en las necesidades de salud (r .537) (Ver tabla 6).

Tabla 6

Correlaciones de Pearson entre variables de responsabilidad social y misión.

\begin{tabular}{|c|c|c|c|c|}
\hline III. Misión & $\begin{array}{l}\text { 6. Enfocan su } \\
\text { servicio social } \\
\text { en la resolución } \\
\text { de problemas de } \\
\text { salud }\end{array}$ & $\begin{array}{l}\text { 7. Incluyen } \\
\text { valores de la } \\
\text { responsabilidad } \\
\text { social en su } \\
\text { misión }\end{array}$ & $\begin{array}{l}\text { 9. Motiva a la } \\
\text { facultad a } \\
\text { participar en } \\
\text { actividades de } \\
\text { responsabilidad } \\
\text { social }\end{array}$ & $\begin{array}{l}\text { 13. Se determinan } \\
\text { los objetivos } \\
\text { de aprendizaje } \\
\text { con base en las } \\
\text { necesidades de } \\
\text { salud }\end{array}$ \\
\hline 48. Se difunde & .454 & $.524^{*}$ & $.549 *$ & .473 \\
\hline $\begin{array}{l}\text { 52. Contribuye } \\
\text { usted a la formación } \\
\text { de profesionales } \\
\text { apegados a los valores } \\
\text { de la facultad }\end{array}$ & $.501^{*}$ & .489 & .440 & $.537^{*}$ \\
\hline
\end{tabular}




\section{Relación entre la percepción de la responsabilidad social que tienen los estudian- tes, el personal académico, directivos, jefes de enseñanza y la formación médica}

Los resultados mostraron que existe relación significativa entre la participación conjunta con otras instituciones en la detección de problemas de salud con el uso del pensamiento crítico para la solución de problemas ( $\mathrm{r}$.533), la identificación del papel del médico en el trabajo interdisciplinario ( $\mathrm{r}$.512) y el desarrollo de protocolos de investigación para la prevención de problemas de salud (r .553).

Se encontró relación significativa entre la inclusión de los principales valores de la responsabilidad social a la misión de la facultad con el uso del pensamiento crítico para la solución de problemas (r .543), la identificación del papel del médico en el trabajo interdisciplinario ( $\mathrm{r}$.629) y el desarrollo de protocolos de investigación para la prevención de problemas de salud (r .621).

También se mostró relación significativa entre la implementación de unidades de aprendizaje de responsabilidad social con el uso del pensamiento crítico para la solución de problemas ( $r$.580), la identificación del papel del médico en el trabajo interdisciplinario (r .613) y el desarrollo de protocolos de investigación para la prevención de problemas de salud (r .576).

Así mismo, se presentó relación significativa entre el apego del programa educativo a la formación de médicos líderes y el uso del pensamiento crítico para la solución de problemas ( $\mathrm{r}$.557), la identificación del papel del médico en el trabajo interdisciplinario (r .578) y el desarrollo de protocolos de investigación para la prevención de problemas de salud (r .560) (Ver Tabla 7).

Tabla 7

Correlaciones de Pearson entre variables de responsabilidad social y formación médica.

\begin{tabular}{lllll}
\hline \multicolumn{1}{c}{$\begin{array}{l}\text { I. Participan } \\
\text { con otras } \\
\text { instituciones } \\
\text { médica } \\
\text { en la detección } \\
\text { de problemas } \\
\text { de salud }\end{array}$} & $\begin{array}{l}\text { 7. Incluyen } \\
\text { los valores de } \\
\text { responsabilidad } \\
\text { social en su misión } \\
\text { social }\end{array}$ & $\begin{array}{l}\text { 16. Implementan } \\
\text { unidades de } \\
\text { aprendizaje de } \\
\text { formación en } \\
\text { responsabilidad } \\
\text { social }\end{array}$ & $\begin{array}{l}\text { 18. Encaminan } \\
\text { su programa } \\
\text { educativo a la } \\
\text { formación de } \\
\text { médicos líderes }\end{array}$ \\
\hline $\begin{array}{l}\text { 29. Utilicen el } \\
\text { pensamiento crítico } \\
\text { para la solución de } \\
\text { problemas }\end{array}$ & $.533^{*}$ & & & \\
$\begin{array}{l}\text { 36. Identifiquen } \\
\text { su papel en la } \\
\text { participación } \\
\text { interdisciplinaria } \\
\text { respetando el } \\
\text { trabajo de los demás }\end{array}$ & & $.543^{*}$ & $.580^{*}$ & \\
\hline
\end{tabular}




\begin{tabular}{|c|c|c|c|c|}
\hline $\begin{array}{l}\text { IV. Formación } \\
\text { médica }\end{array}$ & $\begin{array}{l}\text { 1. Participan } \\
\text { con otras } \\
\text { instituciones } \\
\text { en la detección } \\
\text { de problemas } \\
\text { de salud }\end{array}$ & $\begin{array}{l}\text { 7. Incluyen } \\
\text { los valores de } \\
\text { responsabilidad } \\
\text { social en su misión } \\
\text { social }\end{array}$ & $\begin{array}{l}\text { 16. Implementan } \\
\text { unidades de } \\
\text { aprendizaje de } \\
\text { formación en } \\
\text { responsabilidad } \\
\text { social }\end{array}$ & $\begin{array}{l}\text { 18. Encaminan } \\
\text { su programa } \\
\text { educativo a la } \\
\text { formación de } \\
\text { médicos líderes }\end{array}$ \\
\hline
\end{tabular}

38. Desarrollen

protocolos de investigación para

$.553^{*}$

$.621^{*}$

$.576^{*}$

$.560^{*}$

la prevención de

problemas de salud

\section{Relación entre variables complejas}

En el siguiente diagrama se explica de forma gráfica la relación que existe entre la responsabilidad, la misión y la visión y las variables que lo definen.

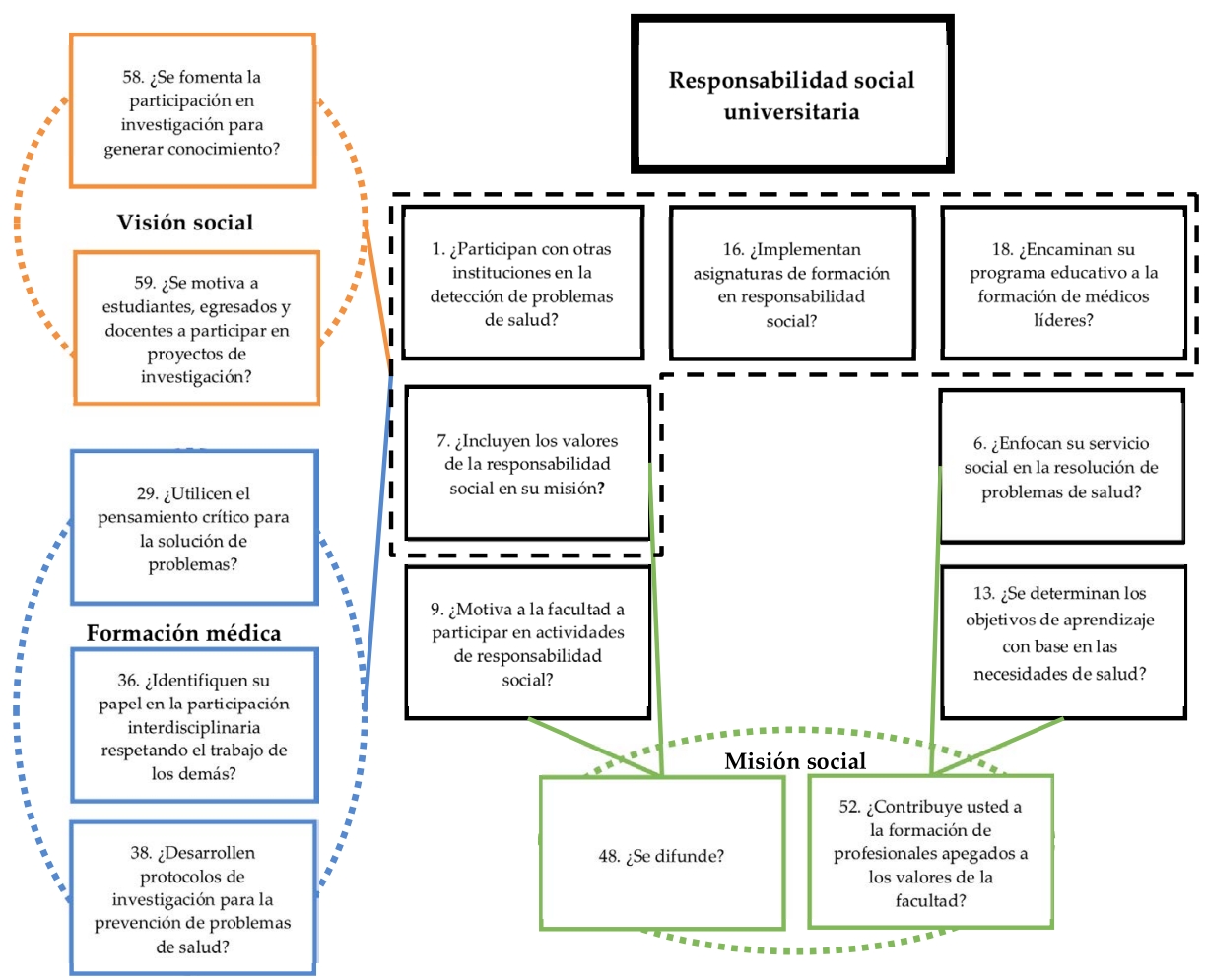

Figura 3. Diagrama de resultados correlacionales. Se pueden observar las relaciones de variables 58 y 59 de la visión social, así como las variables 29, 36 y 38 de la formación médica con las variables 1, 7, 16 y 18 de la RSU. De igual forma se observan las relaciones de las variables 7 y 9 de la RSU con la variable 48 de la misión social, así como la relación entre las variables 6 y 13 de la RSU y la variable 52 de la misión social. 


\section{Discusión y conclusiones}

Esta investigación resaltó que la percepción que tienen de la responsabilidad social, los estudiantes, personal académico, directivos y jefes de enseñanza refiere a un enfoque prospectivo valoral y socioformativo que se orienta a la identificación y solución de problemas de salud de forma colaborativa, con emprendimiento, aplicando procesos metacognitivos y el proyecto ético de vida. Los aspectos mejor percibidos fueron: la implementación de unidades de aprendizaje de formación en responsabilidad social, participación conjunta con otras instituciones en la detección de problemas de salud, inclusión de los valores de la responsabilidad social en la misión social, la orientación del programa educativo a la formación de médicos líderes y la adecuación de proyectos de investigación basados en políticas y reglamentos. Lo cual va más allá de otros enfoques que se tienen de la responsabilidad social como son el consecuencialista y el contractual (Gaete Quezada, 2016). Por lo tanto, percibir a la responsabilidad social desde un enfoque socioformativo, se considera como una aportación relevante de esta investigación.

En lo que respecta a la implementación de unidades de aprendizaje en formación de responsabilidad social, coincide con otra investigación, donde la percepción de la responsabilidad social fue más acertada en grupos de estudiantes de medicina que tomaron cursos enfocados a la responsabilidad social que en los estudiantes que no tomaron estos cursos (Proenço de Oliveira et al., 2019). Según menciona Boelen (2019), la responsabilidad social es el mayor deber social que tienen las escuelas de medicina, por lo que se requiere monitoreo y formación continua (Boelen et al., 2019).

Respecto a la participación con otras instituciones para la detección de problemas de salud, otros estudios destacaron la importancia de la medición de responsabilidad social para lograr adaptar el plan de estudios según las prioridades de la sociedad (Ali et al., 2016). Se encontró una tendencia al crecimiento académico de los egresados y con esto, un sistema de salud basado en especialidades médicas y no en atención primaria (Greer Jr et al., 2017). Esto representa un reto para la educación médica, ya que supone la definición de nuevos roles para los profesionales de la salud. Por lo tanto, es necesario reforzar la red de apoyo en las facultades con la integración de otros actores sociales interesados en que las facultades de medicina tengan un mejor impacto en la salud de la población y en el desempeño del sistema de salud (Boelen \& Woollard, 2011).

La relación que existe entre los aspectos más percibidos de la responsabilidad social y las variables de la visión de la facultad, siendo estas: motivar a estudiantes, egresados y docentes a la participación en investigación para generar conocimiento sobre las necesidades y prioridades de la comunidad; contribuye al mejoramiento de la educación médica basada en la comunidad y los problemas de salud (Ali et al., 2016).

También se encontró relación significativa entre la responsabilidad social y los aspectos más percibidos de la misión de la facultad, los cuales se centraron en la difusión de la misión de la facultad y la formación de profesionales apegados a los valores de la facultad. De acuerdo con Santiago Etxeberria (2012), la formación valoral tiene su sustento tanto en el desarrollo personal, como en la necesidad de adhesión social. Es por esto, que la formación valoral es fundamental para alcanzar el bienestar social (Santiago Etxeberría et al., 2012). 
Igualmente resalta, la relación significativa entre las variables de la formación médica como lo son: el uso del pensamiento crítico para la solución de problemas, identificar el papel del médico en la participación interdisciplinaria y el desarrollo protocolos de investigación para prevenir problemas de salud. Dentro del enfoque prospectivo de las facultades y escuelas de medicina está la identificación de problemas de salud actuales y la previsión de futuros (Preston et al., 2016).

Otro aspecto que llamó la atención en esta investigación fue que los participantes consideraron que, en menor medida, se cuentan con herramientas válidas para la medición del impacto en salud que se tiene en la sociedad. En el análisis comparativo entre estudiantes y personal académico sobre la evaluación periódica de las deficiencias de los servicios de salud resaltó, que el personal académico lo considera menos; asimismo, respecto a los planes de seguimiento de los egresados en la comunidad. Ello coincide con una investigación realizada en México donde algunas facultades de medicina consideran que sus egresados ayudan en gran medida al control de las enfermedades prevalentes; sin embargo, estas afirmaciones no se basan en evidencias medidas con herramientas validadas (Fajardo-Dolci et al., 2019).

Es evidente que la responsabilidad social es tema de gran relevancia, por lo que las instituciones educativas están interesadas en medir el impacto de sus actividades formativas y de sus egresados en la sociedad. Sin embargo, los estudios realizados sobre la percepción de la responsabilidad social, como son: Ali, Fajardo- Dolci, Preston, Shahram y Vallaeys; no establecen relación con la visión, misión y formación médica.

Los aspectos mejor percibidos de la responsabilidad social permiten valorar el conocimiento y comprensión que se tiene de ella, en las facultades y su incidencia en la comunidad universitaria. Lo cual es fundamento para innovar la propuesta curricular con la implementación de unidades de aprendizaje y programas de responsabilidad social.

En este sentido, orientar el plan de estudios a las necesidades de salud, con opciones formativas que promuevan la participación de los estudiantes en programas sociales para la prevención de enfermedades, el desarrollo de protocolos de investigación para la prevención de problemas de salud y el servicio a las comunidades vulnerables; son aspectos clave de la responsabilidad social que se perciben en este estudio.

Por lo que es necesario abordar la responsabilidad social en las unidades de aprendizaje, así como basar el aprendizaje en el servicio a la comunidad y apoyar el desarrollo de proyectos de investigación que tengan impacto en la salud de la sociedad. De igual forma es necesario implementar un modelo de evaluación integral para la mejora continua, que valore la incidencia del desempeño de los estudiantes y la práctica médica de los egresados en la salud de la sociedad.

Es así como esta investigación aporta al estado del conocimiento y contribuye a llenar los vacíos de información de la percepción de responsabilidad social en personal académico y otros actores de las universidades. Asimismo, abre a nuevas líneas de investigación sobre la responsabilidad social como un aspecto articulador del currículo y la sociedad.

\section{Limitaciones}

Este estudio tuvo como limitaciones completar la muestra requerida de alumnos y docentes; solo contestaron 197 estudiantes de los 254 solicitados y 63 de los 67 docentes requeridos, resultado del reacomodo de docentes y estudiantes a la modalidad virtual 
por el confinamiento. Ante ello, se espera que en el futuro este instrumento pueda ser aplicado a una muestra más amplia incluyendo otras facultades, para responder a nuevas líneas de investigación relacionadas con la responsabilidad social.

\section{Reflexiones finales}

El estudio permite conceptualizar a la responsabilidad social de las escuelas de medicina desde un enfoque distinto: prospectivo valoral y socioformativo, que implica formar a los médicos como líderes sociales, acorde a su misión y visión, para que contribuyan a la solución de problemas de salud actuales y a la previsión de problemas futuros de forma colaborativa, con compromiso ético y mediante procesos de investigación inter y transdisciplinarios. El proceso formativo para la responsabilidad social se plantea mediante el diseño de unidades de aprendizaje que incorporen como contenidos a los problemas de salud y como estrategia metodológica el aprendizaje basado en el servicio a la comunidad; lo cual es un aporte a la innovación curricular.

\section{Referencias}

Abreu-Hernández, L. F., Valdez-García, J. E., Esperón-Hernández, R. I., \& OlivaresOlivares, S. L. (2020). El reto de COVID-19 respecto a la responsabilidad social de las escuelas de medicina: nuevas perspectivas profesionales y humanas. Gaceta Médica de México, 156(4), 311-316. https://doi.org/10.24875/GMM.20000306

Ali, E., Hossein, K. M., Mojtaba, M. B., \& Sharareh, K. (2016). An investigation on social accountability of general medicine curriculum. Electronic Physician, 8(7), 2663-2669. http://dx.doi.org/10.19082/2663

Boelen, C., Blouin, D., Gibbs, T., \& Woollard, R. (2019). Accrediting Excellence for a Medical School's Impact on Population Health. Education for Health, 32(1), 41-48. https://doi.org/10.4103/efh.EfH_204_19

Boelen, C., \& Woollard, R. (2011). Consenso Global sobre la Responsabilidad Social de las Facultades de Medicina. Educación Médica, 14(1), 7-14. www.healthsocialac countability.\%0Aorg

Fajardo-Dolci, G. E., Santacruz-Varela, J., Lara-Padilla, E., Martínez, E. G.-L., ZermeñoGuerra, A., \& Gómez, J. C. (2019). Características generales de la educación médica en México. Una mirada desde las escuelas de medicina. Salud Pública de México, 61(5), 648-656. https://doi.org/10.21149/10149

Flórez Guzmán, M. H., Rubio-Rodríguez, G. A., Rodrad Cooperativ, M. S., \& UniveCurvelo Hasssl, J. (2016). Responsabilidad Social Universitaria “Una aproximación desde la percepción de la colectividad académica." Revista Científica Hermes, 17, 80-103. http://www.redalyc.org/articulo.oa?id=477649811005

Gaete Quezada, R. (2016). Percepción de los dirigentes estudiantiles universitarios sobre la responsabilidad social universitaria. Revista de Ciencias Sociales, 11(2), 461-485. https://doi.org/10.14198/OBETS2016.11.2.04

García Ramos, J. M., de la Calle Maldonado, C., Valbuena Martínez, M. C., \& de Dios Alija, T. (2016). La formación en Responsabilidad Social y su impacto en diversas carreras universitarias. Revista de Investigación Educativa, 34(2), 435-451. http://dx.doi. org/10.6018/rie.34.2.244271 
Greer Jr, P. J., Brown, D. R., Brewster, L. G., Lage, O. G., Esposito, K. F., Whisenant, E. B., Anderson, F. W., Castellanos, N. K., Stefano, T. A., \& Rock, J. A. (2017). Socially Accountable Medical Education: An Innovative Approach at Florida International University Herbert Wertheim College of Medicine. Academic Medicine, XX(X), 1-6. https://doi.org/10.1097/ACM.0000000000001811

Hernández, R., Fernández, C., \& Baptista, M. (2014). Metodología de la investigación (Vol. 6, Issue 1, pp. 4-154).

Martínez-Domínguez, L.-M., \& Porto-Pedrosa, L. (2018). Creación del Observatorio de Responsabilidad Social Educativa en América Latina. Revista Iberoamericana de Educación Superior (RIES), IX(26), 212-230. https://doi.org/10.22201/ iisue.20072872e.2018.26.304

Mullan, F. (2017). Social Mission in Health Professions Education Beyond Flexner. JAMA. https://doi.org/10.1001/jama.2017.7286

Preston, R., Larkins, S., Taylor, J., \& Judd, J. (2016). From personal to global: Understandings of social accountability from stakeholders at four medical schools. Medical Teacher, 1-8. https://doi.org/10.3109/0142159X.2015.1114596

Proenço de Oliveira, F., Pacheco Santos, L. M., \& Eri Shimizu, H. (2019). Social Accountability of Medical Schools and Social Representations of Medical Students in the Context of the More Doctors Program. Revista Brasileira de Educação Médica, 43(1), 462 - 472. https://doi.org/10.1590/1981-5271v43suplemento1-20190074.ING

Puschel, K., Riquelme, A., Sapag, J., Moore, P., Díaz, L. A., Fuentes-López, E., Burdick, W., Norcini, J., De La Jara, J. J., Campos, H., Valdez, J. E., Llosa, M. P., Lamus-Lemus, F., Yulitta, H., \& Grez, M. (2020). No Title. Medical Teacher, 42(8), 929-936. https:// doi.org/10.1080/0142159X.2020.1770712

Reeve, C., Woolley, T., Ross, S. J., Mohammadi, L., Halili Jr, S., Cristobal, F., Siega-Sur, J. L. J., \& Neusy, A.-J. (2016). The impact of socially-accountable health professional education: A systematic review of the literature. Medical Teacher, 39(1), 67-73. https:// doi.org/10.1080/0142159X.2016.1231914

Santiago Etxeberría, K., Lukas Mujika, J. F., Lizasoain Hernández, L., \& Joaristi Olariaga, L. (2012). Valores educativos y programas educativos municipales. Revista de Investigación Educativa, 30(2), 367-382. https://doi.org/10.6018/rie.30.2.124951

Shahram, Y., Maryam, A., Mohamed, E. A., Boelen, C., Azizollah, A., \& Hossein, K. M. (2019). Measuring social accountability of medical universities' education functiondesign, development, and validation of instrument. Journal of Evolution of Medical and Dental Sciences, 8(26), 2110-2114. https://doi.org/10.14260/jemds/2019/464

Tobón, S. (2017). Ejes esenciales de la sociedad del conocimiento y la socioformación. In Kresearch (pp. 87-136).

Vallaeys, F., \& Álvarez Rodríguez, J. (2019). Hacia una definición latinoamericana de responsabilidad social universitaria. Educación XX1, 22(1), 93-116. https://doi. org/10.5944/educXX1.19442

Fecha de recepción: 24 de octubre de 2020.

Fecha de revisión: 9 de noviembre de 2020.

Fecha de aceptación: 11 de febrero de 2021. 